\title{
Intestinal biopsy is not always required to diagnose celiac disease: a retrospective analysis of combined antibody tests
}

\author{
Annemarie Bürgin-Wolff', Buser Mauro ${ }^{2}$ and Hadziselimovic Faruk ${ }^{1 *}$
}

\begin{abstract}
Background: The objective of this study was to compare celiac disease (CD)- specific antibody tests to determine if they could replace jejunal biopsy in patients with a high pretest probability of CD.

Methods: This retrospective study included sera from 149 CD patients and 119 controls, all with intestinal biopsy. All samples were analyzed for lgA and IgG antibodies against native gliadin (ngli) and deamidated gliadin peptides (dpgli), as well as for IgA antibodies against tissue transglutaminase and endomysium.

Results: Tests for dpgli were superior to ngli for lgG antibody determination: $68 \%$ vs. $92 \%$ specificity and $79 \%$ vs. $85 \%$ sensitivity for ngli and dpgli, respectively. Positive (76\% vs. $93 \%$ ) and negative (72\% vs. $83 \%)$ predictive values were also higher for dpgli than for ngli. Regarding IgA gliadin antibody determination, sensitivity improved from $61 \%$ to $78 \%$ with dpgli, while specificity and positive predictive value remained at $97 \%(P<0.00001)$. A combination of four tests (IgA anti-dpgli, lgG anti-dpgli, IgA anti- tissue transglutaminase, and IgA anti-endomysium) yielded positive and negative predictive values of $99 \%$ and $100 \%$, respectively and a likelihood ratio positive of 86 with a likelihood ratio negative of 0.00 . Omitting the endomysium antibody determination still yielded positive and negative predictive values of $99 \%$ and $98 \%$, respectively and a likelihood ratio positive of 87 with a likelihood ratio negative of 0.01 .

Conclusion: Antibody tests for dpgli yielded superior results compared with ngli. A combination of three or four antibody tests including IgA anti-tissue transglutaminase and/or IgA anti- endomysium permitted diagnosis or exclusion of CD without intestinal biopsy in a high proportion of patients (78\%). Jejunal biopsy would be necessary in patients with discordant antibody results (22\%). With this two-step procedure, only patients with no CD-specific antibodies would be missed.
\end{abstract}

\section{Background}

Celiac disease (CD) is an immune-mediated enteropathy that is caused by intolerance to gluten in genetically susceptible individuals. Its prevalence among the European population is approximately $1 \%[1,2]$, and is even higher among the elderly [3]. Thus, CD is one of the most frequently occurring lifelong diseases. Serological tests to diagnose $C D$ have improved substantially in the last 20 years. In 1998 [4], we proposed a low-risk and costeffective algorithm to diagnose various forms of gluten-sensitive enteropathy that achieved a positive predictive value (ppv) of $99 \%$, using a combination of

\footnotetext{
* Correspondence: faruk@magnet.ch

${ }^{1}$ Institute for Celiac disease, Bahnhofplatz 11, 4410, Liestal, Switzerland

Full list of author information is available at the end of the article
}

different antibody determinations: anti-endomysium (EMA), IgA anti-tissue transglutaminase (IgA antitTG), and IgA and IgG anti-native gliadin (IgA and IgG anti-ngli). In a population with a high pretest probability of disease, synchronous determination of three or four CD-specific antibodies has a very high ppv and negative predictive value (npv), and may eliminate the necessity of small-bowel biopsy in many patients suspected of having CD [4].

In recent years, the use of ngli as an antigen in antibody-detection tests has been replaced with deamidated gliadin peptides (dpgli), which perform better diagnostically than ngli [5-12]. Our goal in this study was to investigate whether using dpgli instead of ngli, alone or in combination with other tests (EMA and IgA anti-tTG),

\section{Biomed Central}

(C) 2013 Bürgin-Wolff et al.; licensee BioMed Central Ltd. This is an Open Access article distributed under the terms of the Creative Commons Attribution License (http://creativecommons.org/licenses/by/2.0), which permits unrestricted use, distribution, and reproduction in any medium, provided the original work is properly cited. 
reduces the number of jejunal biopsies without missing $\mathrm{CD}$ patients during the diagnostic procedure.

\section{Methods \\ Patients}

Included in this retrospective study were serum samples from 268 patients on a gluten-containing diet. The samples were collected in hospitals or medical services throughout Switzerland, Germany and Austria. The sera were then sent to the Institute for Coeliac Disease in Liestal, Switzerland, where the antibody determinations were performed without any knowledge of each patient's clinical condition. All patients from whom we received a jejunal histology report and clinical data were included in the study. At the beginning of our studies, all patients with symptoms suggestive of $C D$ underwent small intestinal biopsy; antibody determinations were performed at the same time. This diagnostic procedure gradually changed with time as serological tests gained increasing importance in diagnosis, and for an undefined period patients were sometimes selected for biopsy when IgA anti tTG or EMA were positive. The patients suffered from gastrointestinal symptoms such as diarrhea, constipation, poor weight gain, chronic vomiting, abdominal pain, flatulence, and failure to thrive; or disorders such as unexplained weight loss in adults, iron-deficiency anemia, lassitude, psychiatric disorders, short stature, and diabetes type 1. IgA-deficient $\mathrm{CD}$ patients were excluded. Serum for antibody determinations was obtained within 2 months before or one month after endoscopic intervention.

\section{Sample analysis}

All samples were analyzed for antibodies against tTG, ngli, and dpgli by fully automated fluoroenzyme immunoassay tests (Elia Celikey IgA, Elia Gliadin IgA, Elia Gliadin IgG, Elia Gliadin DP IgA and Elia Gliadin DP IgG; Phadia [now Thermo Fisher Scientific], Freiburg, Germany) performed on the Phadia 100 instrument in accordance with the manufacturer's instructions. With the help of ROC curves, we calculated the optimal cutoff values for our sample. For all analyzes except IgA anti-dgpli, we found the best sensitivity and specificity to be consistent with the recommendations of the respective manufacturer. For IgA anti- dpgli. we found that using a cut off value of 7 instead of 10 resulted in somewhat higher sensitivity ( $78 \%$ instead of $71 \%$ ) while maintaining the same specificity.

For each antigen, we determined the best cut-off value within our sample with respect to the sum of falsepositive and false-negative results: $\operatorname{IgA}$ anti $\mathrm{tTG}=7, \operatorname{IgA}$ anti-ngli $=7$, IgG anti-ngli $=7$, IgA anti-dpgli $=7$, and IgG anti-dpgli $=10$. EMA was analyzed by indirect immunofluorescence on monkey esophagus sections: cut-off $=$ serum dilution: 1:5.

\section{Statistical analysis}

In addition to the usual descriptors for diagnostic tests, such as sensitivity, specificity, and positive and negative predictive values, the quantity "efficiency" was used: the efficiency of a diagnostic test is its percentage of correct outcomes. Because predictive values are dependent on the prevalence of the disease in the study population, we also calculated the positive and negative likelihood ratios for $\mathrm{CD}$, which are independent of the pretest probability of disease. The likelihood ratio is the ratio of the probability that a patient with the disease has a particular test result divided by the probability that a patient without the disease has the same test result. Contingency tables were evaluated using "Fisher's Exact Test". To test whether the replacement or addition of a diagnostic test improves the outcome to a statistically significant degree, McNemar's test for significant changes was applied. Both tests were used in their precise form (not only asymptotic) as available in the software package StatXact version 6.3.0. (Cytel Software Corporation Cambridge, MA, USA).

\section{Results}

The histology of 149 consecutive patients (104 females, age range at biopsy $0.9-80$ years, median age 29 years; 45 males, age range at biopsy $2-73$ years, median age 13 years) revealed subtotal or complete villous atrophy, hyperplasia of the crypts, and an increase in intraepithelial lymphocytes (Marsh classification 3a, b, or c lesions). All of these patients recovered after starting a gliadinfree diet and were regarded as having $\mathrm{CD}$. The biopsies of 119 consecutive patients (66 females, age range at biopsy 1.5-72 years, median age 17 years; 53 males, age range at biopsy 2-66 years, median age 7 years) revealed a normal mucosa or a mucosa with slight, nonspecific changes; these patients were considered free of $\mathrm{CD}$ and served as controls (The Control group was younger than the group with $C D, P=0.0074)$.

\section{Deamidated gliadin peptides compared with native gliadins as antigens}

Sera from 149 patients with CD and 119 control patients were tested for IgG and IgA antibodies against dpgli and ngli proteins. IgG antibody determination for dpgli was superior to that for ngli. Specificity was $68 \%$ vs. $92 \%$ and sensitivity was $79 \%$ vs. $85 \%$ for ngli and dpgli, respectively; ppv was $76 \%$ vs. $93 \%$ and npv was $72 \%$ vs. $83 \%$ for ngli and dpgli, respectively. For IgA antibody determination, sensitivity was $61 \%$ vs. $78 \%$ for ngli and dpgli, respectively, while the specificity and ppv remained at a high level of 97\% (McNemar's test for significant changes $P<0.00001$, Table 1). Because dpgli antigens 
Table 1 Antibody tests against deamidated gliadin (dpgli) vs. native gliadin (ngli) in $149 \mathrm{CD}$ patients and 119 controls

\begin{tabular}{|c|c|c|c|c|}
\hline & $\begin{array}{l}\lg \mathrm{A} \\
\text { anti-ngli }\end{array}$ & $\begin{array}{l}\text { IgA } \\
\text { anti-dpgli }\end{array}$ & $\begin{array}{l}\text { IgG } \\
\text { anti-ngli }\end{array}$ & $\begin{array}{l}\text { lgG } \\
\text { anti-dpgli }\end{array}$ \\
\hline Sensitivity & $61 \%$ & $78 \%$ & $79 \%$ & $85 \%$ \\
\hline Specificity & $97 \%$ & $97 \%$ & $68 \%$ & $92 \%$ \\
\hline $\begin{array}{l}\text { Positive predictive } \\
\text { value }\end{array}$ & $97 \%$ & $97 \%$ & $76 \%$ & $93 \%$ \\
\hline $\begin{array}{l}\text { Negative predictive } \\
\text { value }\end{array}$ & $67 \%$ & $78 \%$ & $72 \%$ & $83 \%$ \\
\hline
\end{tabular}

McNemar's test for significant changes $p<0.00001$.

61 correct changes; 154 remain correct; 7 wrong changes; 46 remain wrong.

were clearly superior to ngli, we used only dpgli for further $\mathrm{CD}$-specific antibody determinations.

\section{Antibody profile in $\mathrm{CD}$ and control patients}

We also determined the levels of IgA anti-tTG and EMA in sera from the $149 \mathrm{CD}$ patients and 119 controls (Table 2). Because the IgA anti-tTG and EMA results were comparable, we have omitted the EMA results; instead, we have shown the IgA anti- tTG, and IgA antidpgli, and IgG anti-dpgli antibody levels of each individual and compared them with the histological result. We used a multiple test consisting of three individual tests, which produce a total of eight possible results. We defined the outcome of the multiple tests as positive only when all three individual tests were above the cut-off, and as negative only when all three individual tests were below the cut-off. The majority of the patients (208/268) had either positive (110) or negative (98) results in all three tests. Nearly all patients $(109 / 110)$ who tested positive for antibodies in all three tests had CD according to histological findings. The ppv was $99 \%$ in our population, with a CD frequency of $59 \%$ (Table 3). Patients who did not test positive for CD-specific antibodies in any of the three tests were almost all free of CD according to the results of jejunal biopsy (96/98 patients); the npv was $98 \%$. Patients with discordant antibody results (60/268 patients, $22 \%$ ) could not be defined as positive or negative for $\mathrm{CD}$ with the multiple tests and remained unclassified. The likelihood positive ratio $(\mathrm{lr}+)$ was 87 and the likelihood ratio negative (lr-) was 0.01 (Table 3). These findings indicate that a biopsy is avoidable if all antibody values are either above or below the cut-off. In patients with discordant antibody results, an intestinal biopsy is necessary to diagnose or exclude CD.

\section{Performance of single antibody tests and selected test combinations}

We compared the performance of IgA anti-dpgli, IgG anti-dpgli, IgA anti-tTG, and EMA tests, and calculated the sensitivity, specificity, ppv, npv, lr,$+ \operatorname{lr}-$, and efficiency of each test and some of the possible test combinations (Table 3). We also indicated the absolute number of patients whose antibody test results were falsely positive or falsely negative for $\mathrm{CD}$, as well as those who could not be classified based on antibody tests. Most of the following diagnostic tests are multiple tests (compare with Table 2). We defined the outcome of a multiple test as positive only when all individual tests were above the cut-off, and as negative only when all individual tests were below the cut-off. Test combinations containing only IgA antibodies were not considered; they are unsuitable for diagnostic purposes, because of the possibility that some patients may be deficient in IgA.

Currently, biopsies are often performed when a patient's IgA anti-tTG or EMA test is positive. Negative serological results are usually not followed by a jejunal intervention, unless there is a very strong clinical suspicion of $\mathrm{CD}$. However, the data in Table 3 clearly show that single tests are neither specific nor sensitive enough to reduce the number of biopsies in patients with symptoms of $\mathrm{CD}$, although the number of nonclassified patients was zero. Single tests such as the widely used IgA antitTG test can give rise to many falsely classified patients.

Table 2 Antibody profile in each of 149 CD patients and 119 controls

\begin{tabular}{|c|c|c|c|c|c|c|}
\hline $\lg A$ anti- tTG & IgA anti- dpgli & IgG anti- dpgli & $C D n=149$ & Controls $n=119$ & Total $n=268$ & Classification \\
\hline+ & + & + & 109 & 1 & 110 & 110 positives \\
\hline+ & + & - & 7 & 1 & 8 & \\
\hline+ & - & + & 15 & 4 & 19 & \\
\hline+ & - & - & 13 & 10 & 23 & 60 not \\
\hline- & + & + & 0 & 0 & 0 & classified \\
\hline- & + & - & 0 & 2 & 2 & \\
\hline- & - & + & 3 & 5 & 8 & \\
\hline- & - & - & 2 & 96 & 98 & 98 negatives \\
\hline
\end{tabular}

+ antibody present; - antibody absent. 
Table 3 Performance of single antibody tests and selected combinations $n=268,149$ CD patients and 119 controls

\begin{tabular}{|c|c|c|c|c|c|c|c|c|c|c|}
\hline & fn & $f p$ & nc & sens & spec & ppv & npv & effic & Ir+ & Ir- \\
\hline Single tests & & & & $\%$ & $\%$ & $\%$ & $\%$ & $\%$ & & \\
\hline IgA anti-dpgli & 33 & 4 & 0 & 78 & 97 & 97 & 78 & 86 & 23 & 0.23 \\
\hline lgG anti-dpgli & 22 & 10 & 0 & 85 & 92 & 93 & 83 & 88 & 10 & 0.16 \\
\hline IgA anti-tTG & 5 & 16 & 0 & 97 & 87 & 90 & 95 & 92 & 7 & 0.04 \\
\hline EMA & 3 & 18 & 0 & 98 & 85 & 89 & 97 & 98 & 6 & 0.02 \\
\hline \multicolumn{11}{|l|}{$\begin{array}{l}\text { Combinations of } 2 \text { tests } \\
\text { lgG anti-dpgli }+\end{array}$} \\
\hline IgA anti-tTG & 2 & 5 & 39 & 83 & 82 & 96 & 98 & 83 & 20 & 0.04 \\
\hline IgG anti-dpgli + EMA & 1 & 6 & 39 & 84 & 82 & 95 & 99 & 83 & 17 & 0.01 \\
\hline IgA anti-dpgli + IgG anti-dpgli & 15 & 1 & 37 & 73 & 89 & 99 & 88 & 80 & 87 & 0.11 \\
\hline \multicolumn{11}{|l|}{$\begin{array}{l}\text { Combinations of } 3 \text { tests } \\
\text { IgA anti-dpgli }+\end{array}$} \\
\hline IgG anti-dpgli + EMA & 1 & 1 & 62 & 72 & 81 & 99 & 99 & 76 & 86 & 0.01 \\
\hline IgA anti-dpgli + IgG anti-dpgli + IgA anti-tTG* & 2 & 1 & 60 & 73 & 81 & 99 & 98 & 76 & 87 & 0.01 \\
\hline IgG anti-dpgli + EMA + IgA anti-tTG & 0 & 5 & 45 & 83 & 80 & 96 & 100 & 81 & 20 & 0.00 \\
\hline \multicolumn{11}{|l|}{$\begin{array}{l}\text { Combination of } 4 \text { tests } \\
\text { lgG anti-dpgli }+\end{array}$} \\
\hline IgA anti-dpgli + EMA + IgA anti-tTG & 0 & 1 & 65 & 72 & 79 & 99 & 100 & 75 & 86 & 0.00 \\
\hline
\end{tabular}

*This test combination is identical to the test in Table 2.

Positive, above the cut-off in all tests; negative, below the cut-off in all tests; fn, number of false-negative patients; fp, number of false-positive patients; nc, number of patients not classified because of discordant antibody results; sens, sensitivity; spec,specificity; ppv, positive predictive value; npv, negative predictive value; effic, efficiency; Ir+, likelihood ratio positive; Ir-, likelihood ratio negative.

A combination of two tests also yielded many incorrectly classified patients and is therefore unsuitable for reducing the number of biopsies. The two-test combinations yielded either too many false-positive diagnoses (IgG anti-dpgli + IgA anti-tTG or IgG anti-dpgli + EMA) or too many false-negative diagnoses (IgA antidpgli + IgG anti- dpgli), although the number of nonclassified patients was smaller than in combinations with more than two tests. The combination of four tests was optimal: only one patient was falsely positive, no patients were falsely negative, the ppv was $99 \%$, the npv was $100 \%$, the $\mathrm{lr}+$ was 86 , and the $\mathrm{lr}$ - was 0.00 For practical reasons, a combination of three tests using IgA anti-tTG instead of EMA in combination with IgA anti-dpgli and IgG anti-dpgli (Table 2) may be sufficient to set a standard (ppv 99\%, one false-positive result; npv 98\%, two false-negative results; and $\mathrm{lr}+=87, \mathrm{lr}-=0.01$ ). A biopsy was avoidable in $208 / 268$ patients (78\%), while $60 / 268$ patients $(22 \%)$ could not be diagnosed with the combination of serological tests because their results were in disagreement (only one or two results above the cut-off, with the remaining result(s) below the cut-off; Table 2).

\section{Discussion}

The diagnosis of $\mathrm{CD}$ has traditionally depended upon intestinal biopsies and has been extended to include an array of serological markers. The guidelines of the European and North American societies for gastroenterology require a biopsy for diagnosis $[13,14]$. Recently, the European Society for Pediatric Gastroenterology, Hepatology, and $\mathrm{Nu}$ trition published guidelines allowing the diagnosis of $\mathrm{CD}$ without a biopsy in some situations [15]. CD is usually diagnosed when the duodenal and jejunal mucosa display villous atrophy, crypt hyperplasia, and an increase in intraepithelial lymphocytes [16-19]. However, different diseases not related to gluten- sensitive enteropathy can induce a flat mucosa, thus mimicking CD. Moreover, patients with gluten-sensitive enteropathy and normal small bowel mucosal architecture have also been described [20-24]. Most likely because of a lack of technical proficiency with grasping biopsy forceps or endoscopic procedure, biopsy specimens have been shown to be sufficient for diagnosis of CD in only $90 \%$ of cases [25]. Furthermore, $C D$ may be missed during histological examinations owing to variations in different pathologists' assessments [26]. Because of this, and because of the inconvenience and high cost associated with jejunal biopsy and the high prevalence of $\mathrm{CD}$ in the general population, less-invasive tests are required. In the last 20 years, serological tests for the diagnosis of $\mathrm{CD}$ have improved substantially [27-33]. For practical and ethical reasons, patients with negative serology sometimes did not undergo a biopsy unless clinical indications of $\mathrm{CD}$ were evident. This procedure causes a verification bias because the gold standard (histology of the mucosa) is not always available for negative tests [29]. On the other hand, a 
positive test result demanded a biopsy even when there was only a slight clinical suspicion of CD. Today, it is nearly impossible to overcome this bias for ethical reasons; therefore, the bias may be present in many studies.

The data contained in Table 3, however, indicate that the criteria for choosing the best tests must be defined. For clinicians who want to reduce the number of jejunal interventions in a population with a high frequency of $\mathrm{CD}$, the best test is the one with the lowest sum of falsepositive and false-negative diagnoses: the test with the highest ppv, the highest npv, a high likelihood ratio positive, and a low likelihood ratio negative. In our study, a combination of four antibody tests yielded a ppv of $99 \%$, an npv of $100 \%$, an $1 r+$ of 86 , and an lr- of 0.00 . For practical reasons, we may omit EMA from our combination of antibody tests, and instead chose the test combination of IgG anti-dpgli + IgA anti-dpgli + IgA anti-tTG (Tables 2 and 3), with a ppv of $99 \%$, an npv of $98 \%$, an $\mathrm{lr}+$ of 87 , and an $1 r-$ of 0.01 , as the first step in our diagnostic procedure. Out of 268 patients, 208 (78\%) were correctly classified with these serological tests: they had either three tests above or three tests below the cut- off. Intestinal biopsy was necessary as a second diagnostic step in the remaining 60 patients (22\%), who had discordant antibody results. This twostep diagnostic procedure reduces the number of intestinal biopsies and increases the sensitivity of the entire diagnostic procedure; only $\mathrm{CD}$ patients without any $\mathrm{CD}$ specific antibodies would be missed.

In 1998 [4], we suggested the above combination of serological tests as a low-risk and cost-effective algorithm for diagnosing various forms of $\mathrm{CD}$. This combination - still using anti ngli - was confirmed in a total of 1,873 patients with jejunal biopsy [30-32]. Because of patient preselection according to their symptoms, the prevalence of CD was 59\%. The ppv of three tests with congruent positive antibody results was $>99 \%$. The npv of all three antibody tests was 98\%. However, 37\% (599/ $1,873)$ of the patients with discordant antibody results could not be classified by antibody tests alone. In the present study, the number of nonclassified patients was reduced from $37 \%$ to $22 \%(P<0.001)$ because anti-dpgli performed better than anti-ngli. Thus, in a population with a high pretest probability of $\mathrm{CD}$, using a combination of three or four antibody tests should obviate the need for as many as $78 \%$ of jejunal biopsies.

Several recent studies have questioned the necessity of performing a jejunal biopsy on all individuals with suspected CD [34-39]. One approach defined five criteria, including clinical signs, four of which had to be fulfilled for a diagnosis of $\mathrm{CD}$ [38]. Other studies describe the association of very high IgA anti-tTG antibody titers with Marsh 3 histopathology [35-37]. Therefore, recent guidelines, released by the European Society for Pediatric
Gastroenterology, Hepathology, and Nutrition stated that intestinal biopsy is redundant in patients with high anti-tTG antibody titers ( $>10$ times the upper limit of normal) [15]. These proposals attain a high specificity and will result in few patients receiving a false-positive diagnosis. However, many CD patients do not have such high anti-tTG titer, and will therefore require intestinal biopsy. Sugai et al. [39] investigated single antibody tests and various combinations of two-antibody tests in populations with different pretest probabilities for $\mathrm{CD}$. As in our study, sensitivity was lower in combined tests than in single tests; however, ppv increased significantly not only in the population with high pretest probability for $C D$, but also in the group with low pretest probability for $\mathrm{CD}$. They concluded that: "Appropriate use of $C D$ serology might accurately identify the vast majority of $C D$ patients in populations with different pretest probabilities".

Recently, Vermeersch et al. illustrated the utility of likelihood ratios for the interpretation of $\mathrm{CD}$ serology. [40] The likelihood ratio for $C D$ was much higher for double positive test than for single positive test results. [40] Our results showed comparable test results for single and double positive analyses. (Table 3) Similarly, triple positive tests had a high likelihood ratio. However, the best test for $C D$ exclusion was the triple negative test which had a significantly lower likelihood ratio than the double negative test reults reported by Vermeersch et al. ( $\mathrm{p}=0.000037$ ).

Therefore, we speculate that the combined tests with the very high likelihood ratio positive and the very low likelihood ratio negative achieved in the present study group will also identify patients in populations with a low $\mathrm{CD}$ frequency.

\section{Conclusion}

There is no single test - not even jejunal biopsy - that can conclusively diagnose or exclude $\mathrm{CD}$ in every individual. Therefore, we propose the following two-step diagnostic procedure: The first step is the combined, simultaneous determination of IgA anti-dpgli and IgG anti-dpgli + IgA anti-tTG and/or EMA. The vast majority of patients will have either three positive or three negative results, obviating the need for a biopsy. The second step, jejunal biopsy, should be performed only in patients with discordant antibody results (i.e., in patients whose CD status cannot be classified by antibody tests alone). In any case, effects of a gluten-free diet must be controlled.

\section{Competing interest}

The authors declare that they have no competing interests.

\section{Authors' contributions}

Study design: ABW and FH; Patient selection: ABW and FH; Sample analysis and interpretation: $A B W$ and $F H$; Statistics: MB; Manuscript writing: $A B W, M B$, and $\mathrm{FH}$. All authors have read and approved the final manuscript. 


\section{Author details}

IInstitute for Celiac disease, Bahnhofplatz 11, 4410, Liestal, Switzerland.

${ }^{2}$ Statistik Dr. M. Buser, Riehen, Switzerland.

Received: 13 March 2012 Accepted: 14 January 2013

Published: 23 January 2013

\section{References}

1. Schuppan D, Junker Y, Barisani D: Celiac Disease: From pathogenesis to novel therapies. Reviews in basic and clinical gastroenterology. Gastroenterology 2009, 137:1912-1933.

2. Lohi S, Mustalahti K, Kaukinen K, Laurila K, Collin P, Rissanen H, Lohi O, Bravi E, Gasparini M, Reunanen A, et al: Increasing prevalence of coeliac disease over time. Aliment Pharmacol Ther 2007, 26:1217-1225.

3. Vilppula A, Kaukinen K, Luostarinen L, Krekelä I, Patrikainen H, Valve R, Mäki MCollin P: Increasing prevalence and high incidence of coeliac disease in elderly people: a population-based study. BMC Gastroenterol 2009, 9:49.

4. Hadziselimovic F, Bürgin-Wolff A: Coeliac disease. Lancet 1998, 351:62-63.

5. Aleanzi M, Demonte AM, Esper C, Garcilazo S, Waggener M: Coeliac Disease: Antibody recognition against native and selectively deamidated gliadin peptides. Clin Chem 2001, 47:2023-2028.

6. Schwertz E, Kahlenberg F, Sack U, Richter T, Stern M, Conrad K, Zimmer KP, Mothes P: Serologic assay based on gliadin-related nonapeptides as a highly sensitive and specific diagnostic aid in celiac disease. Clin Chem. 2004, 50:2370-2375.

7. Sugai E, Vazquez H, Nachman F, Moreno ML, Mazure R, Smecuol E, Niveloni S, Cabanne A, Kogan Z, Gomez JC, et al: Accuracy of testing for antibodies to synthetic gliadin-related peptides in celiac disease. Clin Gastroenterology Hepathology 2006, 4:1112-1117.

8. Kaukinen K, Collin P, Laurila K, Kaartinen T, Partanen J, Mäki M: Resurrection of gliadin antibodies in coeliac disease. Deamidated gliadin peptide antibody test provides additional diagnostic benefit. Scand I Gastroenterol 2007, 42:1428-1438

9. Agardh D: Antibodies against synthetic deamidated gliadin peptides and tissue transglutaminase for the identification of childhood celiac disease. Clin Gastroenterol Hepatol 2007, 5:1276-1281.

10. Rashtak S, Ettore MW, Homburger HA, Murray JA: Comparative usefulness of deamidated gliadin antibodies in the diagnosis of Celiac disease. Clin Gastroenterol Hepatol 2008, 6:426-432.

11. Volta U, Granito A, Fiorini E, Parisi C, Piscaglia M, Pappas G, Muratori P, Bianchi FB: Usefulness of Antibodies to Deamidated Gliadin Peptides in Celiac Disease Diagnosis and Follow-up. Dig Dis Sci 2008, 53:1582-1588.

12. Prause C, Ritter M, Probst C, Daehnrich C, Schlumberger W, Komorowski L, Lieske R, Richter T, Hauer AC, Stern M, et al: Antibodies against deamidated gliadin as new and accurate biomarkers of childhood coeliac disease. JPGN 2009, 49:52-58.

13. Report of a working group of the united European gastroenterology week in Amsterdam: When is a coeliac a coeliac? Eur I Gastroenterol Hepatol 2001, 13:1123-1128.

14. Hill ID, Dirks MH, Liptak GD, Colletti RB, Fasano A, Guandalini S, Hoffenberg EJ, Horvath K, Murray JA, Pivor M, et al: Guideline for the diagnosis and treatment of celiac disease in children: recommendations of the North American Society for Pediatric Gastroenterology Hepatology and Nutrition. J Pediatr Gastroenterol Nutr 2005, 40:1-19.

15. Husby S, Koletzko S, Korponay-Szabo IR, Mearin ML, Phillips A, Shamir R, Troncone R, Giersiepen K, Branski D, Catassi C, et al: European Society for Pediatric Gastroenterology, Hepatology and Nutrition Guidelines for the diagnosis of Coeliac disease. JPGN 2012, 54:136-160.

16. National Institutes of Health Consensus Development Conference Statement on Celiac Disease: National Institutes of Health Consensus Development Conference Statement on Celiac Disease. Gastroenterology 2005, 128:1-9.

17. Dickson BC, Streutker CJ, Chetty R: Coeliac disease: An update for pathologists. Review. J Clin Path 2006, 59:1008-1016.

18. Semecoul E, Bay JC: Celiac Disease. WGO-OMGE Practice Guidelines. World Gastroenterology News 2011, 16:7-9.

19. Green PHR, Rostami K, Marsh MN: Diagnosis of Celiac disease (review). Best Pract Res Clin Gastroenterol 2005, 19:389-40.

20. Mäki M, Holm K, Koskimies S, Hällström O, Visakorpi JK: Normal small bowel biopsy followed by coeliac disease. Arch Dis Child 1990, 65:1137-1141.
21. Kaukinen K, Mäki M, Partanen J, Sievänen H, Collin P, Collin P: Celiac disease without villous atrophy. Revision of criteria called for. Dig Dis Sci 2001, 46:879-887.

22. Kurppa K, Collin P, Viljamaa M, Haimila K, Saavalainen P, Partanen J, Laurila K, Huhtala H, Paasikivi K, Mäki M, et al: Diagnosing mild enteropathy celiac disease: A randomized, controlled clinical study. Gastroenterolgy 2009, 136:816-823.

23. Bashir MM, Feighery C, Coates C, O'Shea U, Delaney D, O'Briain S, Kelly J, Abuzakouk M: The absence of a mucosal lesion on standard histological examination does not exclude diagnosis of celiac disease. Dig Dis Sci 2008, 53:52-61.

24. Kaukinen $\mathrm{K}$, Collin P, Mäki M: Latent coeliac disease or coeliac disease beyond villous atrophy? Gut 2007, 56:1339-1340.

25. Collin P, Kaukinen $K$, Vogelsang H, Korponay-Szabó I, Sommer R, Schreier E, Volta U, Granito A, Veronesi L, Mascart F, et al: Antiendomysial and antihuman recombinant tissue transglutaminase antibodies in diagnosis of coeliac disease: a biopsy-proven European multicentre study. Eur J Gastroenterol Hepatol 2005, 17:85-91.

26. Mubarak A, Nikkels P, Houwen R, Ten Kate F: Reproducibility of the histological diagnosis of celiac disease. Scand J Gastroenterology 2011, 1-9.

27. Bürgin-Wolff A, Berger R, Gaze H, Lentze MJ, Nussle D: IgG, IgA and lgE gliadin antibody determinations as screening test for untreated coeliac disease in children, a multicentre study. Eur J Pediatr 1989, 148:5-496.

28. Bürgin-Wolff A, Gaze H, Hadziselimovic F, Huber H, Lentze MJ, Nusslé D, Reymond-Berthet C: Antigliadin and antiendomysium antibody determination for coeliac disease. Arch Dis Child 1991, 66:941-947.

29. Bürgin-Wolff A, Dahlbom I, Hadziselimovic F, Petersson CJ: Antibodies against human tissue transglutaminase and endomysium in diagnosing and monitoring coeliac disease. Scand J Gastroenterol 2002, 6:685-691.

30. Bürgin-Wolff A, Hadziselimovic F: Screening test for coeliac disease. Lancet 1997, 349:1843-1844.

31. Bürgin-Wolff A, Hadziselimovic F: Two-step Approach for Diagnosing Celiac Disease. Clin Gastroenterol Hepatol 2008, 6:1173-1174.

32. Hadziselimovic F, Bürgin-Wolff A: Celiac Disease. N Engl J Med 2008, 358:747-746.

33. van der Windt DA, Jellema P, Mulder CJ, Kneepkens CM, van der Horst HE: Diagnostic Testing for Celiac Disease among Patients with Abdominal Symptoms: A Systematic review. JAMA 2010, 303:1738-1746.

34. Barker CC, Craig M, Garet J, Mock T: Can Tissue Transglutaminase Antibody Titers Replace Small-Bowel Biopsy to Diagnose Celiac Disease in Select Pediatric Populations? Pediatrics 2005, 115:1341-1346.

35. Hill PG, Holmes GK: Coeliac disease: A Biopsy is not always necessary for diagnosis. Aliment Pharmacol Ther 2008, 27:572-577.

36. Donaldson MR, Book LS, Leiferman KM, Zone JJ, Neuhausen SL: Strongly positive tissue transglutaminase antibodies are associated with Marsh 3 histopathology in adult and pediatric celiac disease. J Clin Gastroenterol. $2008 \mathrm{Mar}, 42(3): 256-260$.

37. Mubarak A, Wolters VM, Gerritsen SAM: A biopsy is not always necessary to diagnose celiac disease. J P Gastroenterology Nutrition 2011, 52:554-557.

38. Catassi C, Fasano A: Celiac disease diagnosis: Simple rules are better than complicated algorithms. Am J med 2010, 123:691-693.

39. Sugai E, Moreno ML, Hwang HJ, Cabanne A, Crivelli A, Nachman F, Vazques Z, Niveloni S, Argonz J, Mazure R, et al: Celiac disease serology in patients with different pretest probabilities: Is biopsy avoidable? World I Gastroenterol. 2010 Jul 7, 16(25):3144-3152.

40. Vermeersch P, Geboes K, Marien G, Hoffman I, Hiele M, Bossuyt X: Serological diagnosis of celiac disease: Comparative analysis of different strategies. Clinica Chimica Acta 2012, 413:1761-1767.

doi:10.1186/1471-230X-13-19

Cite this article as: Bürgin-Wolff et al: Intestinal biopsy is not always required to diagnose celiac disease: a retrospective analysis of combined antibody tests. BMC Gastroenterology 2013 13:19. 